\title{
OBSERVATIONS CONCERNING THE CAUSATION OF SIMPLE GLAUCOMA.
}

\author{
JOHN DUNN, M.D., \\ RICHIIONI, VA.
}

\begin{abstract}
This paper explains and upholds the view that simple glaucoma depends upon changes in the vitreous, especially upon swelling of the vitrcous body. Such essential condition is not removed by treatment that may give temporary relief from the more prominent symptoms.
\end{abstract}

Simple glaucoma is an ocular state which results from swelling of the vitreous body. Closure of the Fontanic drainways is no more its cause than is cupping of the optic disc. Both of these pathologic entities are the immediate results of the swelling of the vitreous; each is responsible for its own set of symptoms, which in turn are a part of the complete syndrome. Swelling of the vitreous body is to be compared with swelling of the crystalline lens. Both have the same causes, socalled age changes or an as yet undetermined state of the blood. As in the lens, so in the vitreous, the degree of the swelling is limited by the nature of the body and the processes which produce it. The actual possible increase in both is small. Moreover, simple glaucoma is bilateral, and, as a rule, does not appear prior to the time of life when the socalled presbyopic changes have occurred in the crystalline lens. Such is my belief in regard to the nature of simple glaucoma, and the more I reflect upon the problem the clearer its details become.

It was my fortune to have under my care within a few months thirty or more eyes affected with either simple glaucoma, or with simple glaucoma to which was superadded an acute phase. Each case was the cause of much reflection on my part, and as those cases succeeded one another they only served to make clearer the conviction as to the nature of this truly to be dreaded ocular disease. In the discussion to follow, I must admit at the outset that I have no microscopic proof of my belief; nor have I been able to devise a plan by which, with the limited laboratory facilities at my command, I can produce such proof. My deductions were obtained by envisaging each case as it came and by attempting to ex- plain the conditions present in each by one or the other of the many theories which ophthalmology has suggested. When finally I came to the conclusion that swelling of the vitreous body and that this alone is the cause of simple glaucoma, I could not recall that I had ever seen in my medical readings mention of the posibibility of such a process affecting the vitreous. Nor have I since seen this mentioned as the cause of the disease under consideration.

Fluidity of the vitreous is a well recugnized condition. It varies in degree. It is coexistent with a normal, subnormal or increased intraocular tension, as measured with the tonometer. Its pathology must consist in the disappearance of certain of the vitreous elements and their replacement, in so far as the condition of the ocular tissues, including that of the ciliary processes, will admit, by the aqueous fluid. Increased intraocular tension as the result of increase in the amount of the aqueous within the eye is well recognized c.gr. in cases of buphthalmos. Here we have congenital absence of the essential elements of the Fontanic system. This, however, is not simple glaucoma.

I am rather inclined to think that contemplation of the pathology of the congenital type of socalled glaucoma has hat much to do with the misappreciation of what goes on in simple glatcoma. These two types of glaucoma are of entirely different pathology. As I see it, the one shows increased intraocular tension with increased volume of retained aqueous as the result of $a b-$ sence of the essentials of the Fontanic channels; the other shows increased intraocular tension primarily as the result of swelling of the elements of the vitreous substance. The higher than normal tension here is increased by 
blockage. from pressure of the vitreous, on the exits in the Fontanic system. The increase in the amount of excess aqueous in the anterior chamber is sniall. The anterior chamber has little tendency to deepen and for two reasons: (1) The aqueous is as incompressible as the vitreous. (2) The ocular envelopes can withstand evenly distributed pressure from within higher than the glaucomatous process develops. (The local exception to this statement is found in the lamina cribrosa).

It has often been noted that simple glaucoma may exist in the presence of either a deep or shallow anterior chamber. The apparently deep anterior chamber is not deep because of the increased amount of aqueous therein. I have never seen a case where the depth of the anterior chamber was greater than is to be seen from time to time in normal cyes. We have been accustomed to expect a shallow anterior chamber in glaucoma of the acute inflammatory type, and when we see a deep chamber in any type of glaucoma it is a matter of mental comment. The shallow chamber in simple glaucoma may be only a normally shallow chamber, or it may represent a relaxation of the suspensory ligament of the lens as the result of the pressure of the vitreous plus sudden inequalities of escape of the aqueous.

In bupthalmos retention of the aqueous results in increase in the size of the whole ball, not only of the anterior chamber-in this disease the aqueous is forced thruout the vitreous substance; the yielding of the ocular tunics is the result of the fact that the tissues of the eycball lack the resisting properties which later in life they acquire. Increase in size of the vitreous is followed in the course of time by liquefaction of the vitreous. In what does liquefaction of the vitreous consist? In the disappearance of certain essential elements of the vitreous body and their replacement by the secretions from the ciliary processes, i.e., the aqueous.

Why, then, does not the high tension of simple glaucoma disappear after partial liquefaction of the vitreous sets in, if simple glaucoma be due to swelling of the vitreous elements, or, again, if simple glaucoma be due primarily to a swollen vitreous, why cannot a normal or subnormal tension be maintained after removal of a part of the vitreous? I think the answer to these two questions is to be sought in the pressure effects of the swollen vitreous in its cnveloping membrane on the channels of exit in the Fontanic angle. The months long, or years long unrelieved pressure results in a proportionate obliteration of these drainways. (An argument for early operative intervention). For a considerable, probably a long time after the processes which result in swelling of the vitreous clements have set in, the discomforts they cause are too vague to be referred by the patient to their proper source. The result is, when the patient comes for examination the oculist is confronted by an old process, one that in every case has resulted in marked obliteration of the ultimate channels of exit, most frequently by cupping of the papilla, in many cases by a varying degree of liquefaction of the vitreous body. Bearing these things in mind, we can see that the success of our operative efforts will be measured by the degree of our success in reopening the closed Fontanic channels.

Thus, for example, in cases where the swelling of the vitreous has never been great enough to cause a tension above 38 or 40 , where for the greater part of the time since the glaucomatous process has set in, the tension has remained below 35 , where the eyeball is relatively large, where it has never been subjected to unrelicved subacute or acute exacerbations, a large peripheral iridectomy will, as a rule, (even tho the tension be only slightly reduced as a result of the operation) prevent for a long time (I have known it to do so for twelve years) further demonstrable deterioration of the vision or visual field, and this in the presence of a glaucomatous disc. What an iridectomy has accomplished is the removal of a part of the solid portions of the intraocular structures. This permits a readjustment of the lines of pressure of the swollen 
vitreous and the partial reopening of the as yet not permanently closed channels of exit.

Where, however, the swelling of the vitreous is so great as to result in a very high intraocular tension, and this has persisted so long as to permanently glue together the minute channels of exit, an iridectomy, no matter how well executed or how large, will fail to permanently reduce the tension. With these facts in mind we can see why in one case an iridectomy fails and in another is a success.

In considering what takes place when the vitreous body swells, we must bear in mind the impermeability of its limiting membrane; witness, for example, the picture seen in thrombosis of the central retinal vein or its major branches. Here in spite of marked local retinal edema, with excessive bending of the venous branches and the concurrent hemorrhages, we find no edema or blood in the vitreous. There is nothing in the clinical pictures or histories of the well known inflammatory conditions affecting the cilio-corneal region which lends itself to the belief that simple glaucoma is of inflammatory origin. Where in acute iridocyclitis, as is occasionally the case, the intraocular tension rises above the normal, there are pain or redness and external and internal visible conditions which are never seen in uncomplicated simple glaucoma. In simple glaucoma, for a long time after the process sets in, there is no visible trace of external congestion of the sclera or conjunctiva about the corneal ring; the pupil reacts normally; the range of accommodation is not demonstrably lessened; the vision remains normal; the field of vision remains uncontracted; indeed, were there not means of detecting the existence of a higher than normal intraocu'lar tension, we should be left with the indefinite nervous symptoms, including headache and aching of the cyeballs which follow attempts to use the eyes for close work, as the only symptoms we could detect. In this stage of the disease, we are faced with a problem whose only symptoms are noninflammatory rise of intraocular tension and cyestrain. The eyestrain is manifestly the result of the increased tension; therefore we have to seek a cause for only one thing; viz., increased tension. This must come from one of two causes, increase in size of the vitreous body or noninflammatory closure of the Fontanic exits.

Now, whatever our hypothesis may be, it must have as its basis a terminal condition, for primary glaucoma having once been established continues thru life. It is an incurable as are the age changes in the crystalline lens. It cannot originate in loss of function of the iris or ciliary movements, or atrophy of the ciliary glandular elements, for these continue to function until atrophic degeneration from pressure sets in. I know of nothing which would properly suggest paralysis or disturbance of the sympathetic control of the cilio-glandular system as a possible cause. Section of the sympathetic does not result in primary glaucoma. Swelling of the lens cannot be the cause, for simple glaucoma occurs and may last a long time without any demonstrable refractive changes. As far as I can see it then, we must look for changes in the vitreous body as the cause of simple glaucoma.

That the crystalline lens swells or diminishes in size as the result of what we term age changes is well known. That the vitreous body tends in old age to develop changes which result in fluidity is also a well recognized chapter of its life history. That a possibility exists that it may undergo changes which result in its general enlargement from swelling of its essential elements must at least be admitted. That these changes may be followed later by atrophy of the hypertrophied or swollen elements with a resultant liquefaction of the vitreous is highly probable. That changes similar to those which occur in simple glaucoma take place in the vitreous of all eyes in the later years of life is to be admitted. The degree of these changes determine the presence or absence of simple glaucoma. That other causes than age changes may result in swelling of the vitreous elcments is probable, but 
whether this be the result of certain endocrin insufficiencies or of toxins inthe blood, I cannot say. Probably the former is the correct answer.

If then we grant that swelling of the elements of the vitreous body is a pathologic possibility, we have a solution of the cause of simple glaucoma. In reading what is now immediately to follow, we must bear in mind that we have confined our comments entirely to those stages of simple glaucoma which precede the irritative or inflammatory complications, for these latter are rendered possible as a result following the pressure of the enlarged vitreous, and are in no sense responsible for the primary swelling of the vitreous. The pressure of the swollen vitreous is exerted equally in all directions. Its pressure posteriorly results in forcing back. wards of the lamina cribrosa with a resultant cupping of the disc, which in turn forces against the edge of the cup the retinal fibers and their blood vessels. As a result of this we have (1) atrophy of the retinal fibers with changes in the visual field; (2) compression of the arteries and veins, which produces circulatory disturbances in the retinal vessels, which are especially noteworthy where, as often is the case, these vessels are the seat of sclerotic changes. The lateral pressure of the swollen vitreous is most noticeable when it is sufficiently high to obstruct wholly or partially the exits of the venae vorticosae, and is then represented visibly by the dilatation of the middle episcleral veins. Closure of the exits of the venae vorticosae in itself is followed by increased intraocular tension, but where this occurs as the result of swelling of the vitreous we must bear in mind that the simple glaucomatous condition is not the result of the obstruction of the exits of the venae vorticosae but of swelling of the vitreous itself. The anterolateral pressure is exerted against the Fontanic area, i.e., the sclerocorneal region. This produces flattening of the ciliary processes and proportionate closing of the channels in the Fontanic system, with a forcing forwards and outwards of the root of the iris.
That the closure of the ultimate channels of exit for the aqueous is for a long time not the result of the presence of fibrinous deposit therein. but is solely the result of pressure, is proven by the fact that the use of eserin is so often capable of producing a marked reduction of the intraocular tension. Were the channels blocked with a fibrinous deposit, eserin could produce no such result. (Ilere we have a test which will help us to decide for or against the use of iridectomy in many cases.) Where the pressure of the swollen vitreous has been great (for the degree of swelling of the vitreous varies in different eyes) and has lasted for a considerable time, the re-ult is permanent closure of so great a proportion of the Fontanic channels of exit, that the use of eserin does not reduce the intraocular tension, nor will iridectomy afford relief. Again, there is clinical evidence that sclerocorneal trephining is often followed by a gradual opening of a part of the closed channels of exit. This is to be expected. Lastly we have the picture presented in simple glaucoma in those cases where in the presence of high tension the lamina has not yet given way. The pressure of the vitreous against the retina produces an amblyopia without any contraction of the visual fields. Here at first the amblyopia is transient, lasting for periods of varying duration, and recurring at intervals of different length. I have scen in one case, notable among the cases under my care, the vision increase from $18 / 200$ to $18 / 15$ in a few moments after the instillation of eserin.

A few words must be added about the irritative and inflammatory stages of simple glaucoma, and I think much of the confusion which exists in regard to the causation of this disease is due to the fact that these complications have not always been clearly recognized as ore of the results of the increased intraocular pressure, as much so as cupping of the disc or blockage of the Fontanic channels. In our treatment of simple glaucoma we must bear clearly in mind that doing away with the ir- 
ritative or inflammatory symptoms does not cure the disease. In the presence of the pressure exerted by the swollen vitreous against the cilio-iridic recrion, the ciliary muscle readily tires (witness the easily produced eyestrain of the simple glaucomatous eye). This functional exhaustion disposes the ciliary region to active congestion, if the blood contains an undue proportion of toxins from foci of infection. This congestion may be slight or severe and hence the irritative or the socalled inflammatory types. Remozal of the foci of infection, if done in time, is followed by disappearance of the irritative or inflammatory stage, but the process known as simple glaucoma remains present as before. We have in the above the explanation of the socalled cures of acute glaucoma following extraction of a tooth, or opening and draining of a nasal air chamber, which from time to time have been reported in medical literature.

\title{
CLINICAL OBSERVATIONS ON INCREASED INTRAOCULAR TENSION.
}

\author{
Thomas Faith, M.D., \\ CHICAGO, IILLINOIS.
}

The differentiation between glaucoma and uveitis with increased intraocular tension is sometimes very difficult, altho text-books fail to point this out. This paper reports a series of cases in which the tension was reduced to normal and permanent cure secured by the use of mydriatics. Read before the Chicago Ophthalmological Society, February 20, 1922. See p. 379 .

It is not the intention of this paper to open a general discussion on the subject of glaucoma, but to review a study of the following cases which will illustrate the difficulty frequently encountered in differentiating some of the cases of uveal inflammation which come under observation from cases of primary glaucoma. If we read either a modern text-book on ophthalmology, or one published twenty years ago, the conclusion arrived at will be that the two conditions-iridocyclitis and glaucoma-have well marked differential points, which enable one to make a diagnosis at once. Yet this is a question which often grives not only the student but also the trained ophthalmologist the most concern in practice. Time and again this question presents itself, and we are all called upon to make a decision which is difficult as well as extremely important.

Classical cases are, of course, readily recognized, but the atypical cases are the difficult as well as the interesting ones. We are all aware that every eye which has increased intraocular tension is not affected with glaucoma, and we should keep this fact firmly fixed in our minds, since it often happens that increased hardness of the eyeball is such an outstanding feature in the case under observation that it scems to engage our entire attention, even to the exclusion of all other symptoms, and primary glaucoma is our first and only thought; and following on in this direction miotic treatment is at once resorted to, sometimes with disasterous results, whereas mydriatic treatment would have saved the day. The following cases are illustrative of this point and are submitted for that purpose.

Case 1. Many years ago a young ma11 22 years of age came under my observation, who had been suffering with severe pain in the left eye for a number of days. The jain was so severe when I first saw him that he was nauseated and had been vomiting most of the time during the previous days. I found his pupil partly dilated, the anterior chamber practically obliterated, eyeball hard, and the lens somewhat opaque. Ile had been struck on the eye by the ramrod of a toy pistol about twelve days previously. There was apparently no rupture of the anterior capsule of the lens and no lens matter could be seen in the anterior chamber.

I reasoned that the capsule had been ruptured posteriorly and that the lens swelling had pushed the iris forward 